\title{
Long-term outcomes of artificial chordal replacement with tourniquet technique in mitral valve repair: A single-center experience of 700 cases
}

\author{
Minoru Tabata, MD, MPH, ${ }^{\mathrm{a}}$ Hitoshi Kasegawa, MD, ${ }^{\mathrm{a}}$ Toshihiro Fukui, MD, ${ }^{\mathrm{a}}$ Atsushi Shimizu, MD, \\ Yasunori Sato, $\mathrm{PhD},{ }^{\mathrm{b}}$ and Shuichiro Takanashi, $\mathrm{MD}^{\mathrm{a}}$
}

\begin{abstract}
Objective: Artificial chordal replacement has been shown to be effective and durable, with numerous techniques reported. However, the outcomes of each technique have remained poorly defined. We report the long-term outcomes of the tourniquet technique.
\end{abstract}

\begin{abstract}
Methods: We reviewed the data from 700 patients who had undergone mitral valve repair with the tourniquet technique from 1992 to 2010 . We analyzed the operative outcomes, long-term survival rate, freedom from reoperation, and freedom from recurrent moderate or severe mitral regurgitation (MR). We also performed Cox regression analysis to explore the predictors of recurrent MR after mitral valve repair using the tourniquet technique.
\end{abstract}

\begin{abstract}
Results: The mean age was $54.7 \pm 14.9$ years; 212 patients $(30.3 \%)$ had anterior leaflet prolapse, $142(20.3 \%)$ had posterior leaflet prolapse, and $346(49.4 \%)$ had bileaflet prolapse. Operative mortality was $1.3 \%$. In 26 cases $(3.7 \%)$, mitral valve repair was unsuccessful and was converted to replacement. Of those successfully repaired, the 12-year survival rate, freedom from mitral reoperation, freedom from recurrent moderate or severe MR, and freedom from recurrent leaflet prolapse was $85.9 \%, 88.7 \%, 72.3 \%$, and $89.0 \%$, respectively. The significant predictors of recurrent MR were anterior leaflet prolapse, age, New York Heart Association class III or IV, left ventricular end-systolic dimension, no annuloplasty ring or band, and postoperative residual mild or greater MR.

Conclusions: The tourniquet technique is a simple and effective method to repair leaflet prolapse, with a low incidence of recurrent prolapse. The incidence of recurrent MR was high in the anterior leaflet prolapse group. Age, no annuloplasty ring or band, and residual MR were strong predictors of recurrent MR. (J Thorac Cardiovasc Surg 2014;148:2033-8)
\end{abstract}

Supplemental material is available online.

Mitral valve repair has been the reference standard therapy for severe degenerative mitral regurgitation (MR). Artificial chordal replacement using expanded polytetrafluoroethylene (ePTFE) sutures was introduced in the $1980 \mathrm{~s}^{1}$ and has been widely performed since then. The decision regarding the length of the artificial chordae will be critical

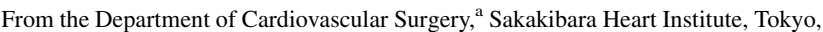
Japan; and Clinical Research Center, ${ }^{\mathrm{b}}$ Chiba University Graduate School of Medicine, Chiba, Japan.

Disclosures: Dr Tabata reports lecture fees from Edwards Lifesciences. Dr Sato reports consulting fees from Kowa Soyaku, Siemens AG, and Pfizer and lecture fees from Teijin. All other authors have nothing to disclose with regard to commercial support.

Received for publication Aug 9, 2013; revisions received Feb 28, 2014; accepted for publication March 21, 2014; available ahead of print April 24, 2014.

Address for reprints: Minoru Tabata, MD, MPH, Department of Cardiovascular Surgery, Sakakibara Heart Institute, 3-16-1 Asahi-Cho, Fuchu-Shi, Tokyo 183-0033, Japan (E-mail: mtabata@ post.harvard.edu).

$0022-5223 / \$ 36.00$

Copyright $(2014$ by The American Association for Thoracic Surgery

http://dx.doi.org/10.1016/j.jtcvs.2014.03.045
}

for successful repair, and numerous techniques have been developed to determine the appropriate length of the artificial chordae. Although artificial chordal replacement has been shown to be effective and durable, the outcomes of each technique have not been well reported.

The tourniquet technique was introduced by Kasegawa and colleagues ${ }^{2}$ in 1992 as a simple method to determine the artificial chordal length. We have been using this technique for artificial chordal replacement since then. In the present study, we analyzed the early and late outcomes of mitral valve repair using the tourniquet technique and evaluated the effectiveness and durability of this technique. We also explored the predictors of recurrent MR after mitral valve repair using this technique.

\section{METHODS}

\section{Study Design and Patient Data}

The present study was a retrospective cohort study. The institutional review board of Sakakibara Heart Institute approved the study and waived the requirement for informed consent.

From January 1992 to December 2010, 1112 adult patients underwent mitral valve repair for type II MR at our institution. Of the 1112 patients 700 underwent mitral valve repair using the tourniquet technique. We excluded pediatric patients $<18$ years old at surgery from the present study. 


$$
\begin{aligned}
& \text { Abbreviations and Acronyms } \\
& \begin{aligned}
\mathrm{CI} & =\text { confidence interval } \\
\mathrm{ePTFE} & =\text { expanded polytetrafluoroethylene } \\
\mathrm{HR} & =\text { hazard ratio } \\
\mathrm{MR} & =\text { mitral regurgitation } \\
\mathrm{TTE} & =\text { transthoracic echocardiography }
\end{aligned}
\end{aligned}
$$

We reviewed the perioperative data and postoperative follow-up information.

The outcome measures included operative mortality, intraoperative conversion to mitral valve replacement, residual mild or greater MR on the predischarge transthoracic echocardiogram (TTE), long-term survival, freedom from mitral reoperation, freedom from recurrent moderate or severe MR, and freedom from recurrent mitral leaflet prolapse. All longterm and echocardiographic outcomes were analyzed, excluding 26 patients who had undergone mitral valve replacement in their primary surgery. Operative mortality was defined as death within 30 days after surgery or before discharge. Survival was calculated from surgery until death, and patients alive were censored at the point of last contact. The mean followup period was $80 \pm 55$ months after surgery, and $94 \%$ of patients had complete follow-up data available.

\section{Operative Technique and Intraoperative TEE}

Surgery was performed through a median sternotomy or right minithoracotomy. All surgery was performed using cardiopulmonary bypass and cardioplegic arrest. The mitral valve was accessed through a superior transseptal approach in median sternotomy cases and through a standard left atriotomy in minithoracotomy cases. Mitral annuloplasty using a ring or band was routinely performed; however, a few patients did not undergo mitral annuloplasty in the early study period. Posterior leaflet prolapse was primarily repaired with leaflet resection with or without sliding valvuloplasty, and artificial chordae were added as a supplementary procedure, if necessary. In this cohort, only a few patients underwent artificial chordal replacement without resection of the posterior leaflet. Anterior leaflet prolapse was primarily repaired with artificial chordal replacement using the tourniquet technique.

To implant artificial chordae, we first fixed CV5 ePTFE sutures (L. Gore $\&$ Associates, Inc, Flagstaff, Ariz) on the correspondent papillary muscle head in a pledgeted mattress fashion. Next, we passed 2 ends of the suture through the prolapsing segment of the leaflet. After seating the annuloplasty ring or band, we placed small tourniquets on each pair of artificial chordae, held them at a specific length by approximating the normal adjacent chordae, and assessed the leaflet competency by injecting saline into the left ventricle (Figure E1). We monitored the left ventricular volume using TEE and filled the left ventricle to the approximate end-systolic volume. If the saline testing showed poor competency, we would readjust the length by sliding the tourniquet and reassess the leaflet competency with saline testing. Once we had confirmed good competency, we gently clamped the ePTFE sutures just below the lower end of the tourniquet with a right-angled clamp, removed the tourniquets, and tied the sutures. We used a very thin and light tourniquet tube for this technique (Figure E2). The tourniquet tube we have used is $1.0 \mathrm{~mm}$ in inner diameter, $1.7 \mathrm{~mm}$ in outer diameter, and $1 \mathrm{~g}$ in weight. If the tourniquet is too thick or too heavy, it will deform the valve during saline testing, making it difficult to assess leaflet competency.

After the repair, the mitral valve was evaluated using intraoperative TEE. Repeat repair or mitral valve replacement with chordal preservation was performed at the same operation if the intraoperative TEE showed significant residual MR after repair. For quick screening of residual MR, the MR jet area was measured. Our indication for a second cardiopulmonary bypass pump run for repeat repair or replacement was $>2.0 \mathrm{~cm}^{2}$ of MR jet area before 2006 and $>1.0 \mathrm{~cm}^{2}$ since 2006 .

\section{TEE Study}

TTE studies were performed before surgery, before discharge, and during follow-up. The mean TEE follow-up period was $64 \pm 56$ months, and 662 patients $(98.2 \%)$ underwent $\geq 1$ TTE after discharge. We reviewed 3424 TEE studies. The left ventricular ejection fraction was calculated using the biplane Simpson method. The left ventricular end-systolic dimension was measured from the 2-dimensional parasternal long-axis view. An effective MR orifice area was calculated as the ratio of MR flow to regurgitant velocity using the proximal isovelocity surface area method. The MR grade was quantitatively determined according to the American College of Cardiology/American Heart Association 2006 guidelines. $^{3}$ When the quantitative assessment of MR was not applicable, MR severity was determined semiquantitatively using other parameters such as the vena contracta and MR jet area. ${ }^{4}$ Residual MR was defined as mild or greater MR on the postoperative predischarge TTE. Recurrent MR was defined as moderate or severe MR on any TTE after surgery.

\section{Statistical Analysis}

Continuous variables are expressed as the mean \pm standard deviation if normally distributed or as the median otherwise. For the time-to-event outcomes (survival, cardiac adverse event-free rate, and freedom from recurrent MR), the interval to a first event was compared using the log-rank test, and the Kaplan-Meier method was used to estimate the absolute risk of each event for each group. Stepwise Cox regression models were used to explore the predictors of recurrent MR. We included the following variables in the model: anterior leaflet prolapse, posterior leaflet prolapse (bileaflet prolapse was the reference for prolapse location), age, hypertension, atrial fibrillation, New York Heart Association class III or IV, a history of endocarditis, ejection fraction, left ventricular end-systolic dimension, no annuloplasty ring or band used, and postoperative residual MR. The hazard ratios (HRs) and 95\% confidence intervals (CIs) were estimated. All comparisons were planned, and the tests were 2-sided. All statistical analyses were performed by using PASW 18.0 (SPSS Inc, Chicago, Ill).

\section{RESULTS \\ Preoperative Characteristics}

The mean age of all patients was $54.7 \pm 14.9$ years, and 230 patients $(32.9 \%)$ were women. Isolated anterior leaflet prolapse was present in 212 patients $(30.3 \%)$, isolated posterior leaflet in $142(20.3 \%)$, and bileaflet prolapse in 346 patients $(49.4 \%)$. The preoperative characteristics of the patients are listed in Table 1 .

\section{Operative Results}

The operative data are listed in Table 1. In the present cohort, 661 cases $(94.4 \%)$ ) were performed by way of a median sternotomy and 39 cases (5.6\%) using a right minithoracotomy. Concomitant cardiac procedures were performed in 260 patients $(37.1 \%)$. For mitral valve repair, we performed artificial chordal placement using the tourniquet technique in all cases, and the median number of leaflet segments in which artificial chordae were placed was 2 segments (range, 1-7). We also performed concomitant mitral annuloplasty with a ring or band in 680 patients $(97.1 \%)$, and leaflet resection with or without sliding valvuloplasty in 473 patients $(67.5 \%)$. We repeated the cardiopulmonary 
TABLE 1. Preoperative characteristics of patients and operative data $(\mathbf{n}=700)$

\begin{tabular}{lc}
\hline \multicolumn{1}{c}{ Variable } & Value \\
\hline Mean age (y) & $54.7 \pm 14.9$ \\
Women & $230(32.9)$ \\
NYHA class III or IV & $230(32.9)$ \\
Atrial fibrillation & $120(17.1)$ \\
Hypertension & $217(31.0)$ \\
History of endocarditis & $81(11.6)$ \\
Emergency surgery & $7(1.0)$ \\
Mean LVEF (\%) & $65.1 \pm 9.0$ \\
Mean LVESD (mm) & $35.4 \pm 4.9$ \\
Anterior leaflet prolapse & $212(30.3)$ \\
Posterior leaflet prolapse & $142(20.3)$ \\
Bileaflet prolapse & $346(49.4)$ \\
Concomitant procedure & $260(37.1)$ \\
$\quad$ Aortic valve replacement & $12(1.7)$ \\
Tricuspid valve repair & $140(20.0)$ \\
$\quad$ Ablation surgery & $114(16.3)$ \\
Coronary artery bypass grafting & $45(64.3)$ \\
Thoracic aortic surgery & $12(1.7)$ \\
Median number of leaflet segment repaired with artificial & $2(1-7)$ \\
$\quad$ chordae & \\
Concomitant mitral valve procedures & $698(99.7)$ \\
Leaflet resection with or without sliding valvuloplasty & $473(67.6)$ \\
Mitral annuloplasty using a ring or band & $680(97.1)$ \\
Repeated cardiopulmonary bypass runs for residual MR & $62(8.9)$ \\
Conversion to mitral valve replacement & $26(3.7)$ \\
\hline Data presented as mean \pm standard deviation, median (range), or n $(\%) . N Y H A, N e w$ \\
York Heart Association; $L V E F$, left ventricular ejection fraction; $L V E S D$, left ventric- \\
ular end-systolic dimension; $M R$, mitral regurgitation. & \\
&
\end{tabular}

bypass runs to fix residual MR during the same surgery in 62 patients $(8.2 \%)$. We converted the procedure to mitral valve replacement for failed repair in 26 patients $(3.7 \%)$. The reasons of conversion were residual MR that was difficult to repair in 22 patients, systolic anterior motion in 2, papillary muscle rupture in 1 , and left ventricular rupture in 1 patient.

The operative mortality was $1.3 \%$ ( 9 of 700 ). Six operative deaths occurred in those undergoing mitral valve repair. The cause of death for those 6 patients was respiratory complications in 3, postcardiotomy cardiogenic shock in 1 , stroke in 1 , and sepsis in 1 . Three operative deaths occurred in cases converted to mitral valve replacement. The cause of deaths for those 3 patients was left ventricular rupture, postcardiotomy cardiogenic shock, and ventricular arrhythmia in 1 each.

\section{Early TTE Findings}

All patients had undergone a postoperative predischarge TTE. Excluding the 26 patients who had undergone conversion to mitral valve replacement, 82 patients $(12.2 \%)$ had mild or greater MR. The incidence of residual MR decreased dramatically from $24.4 \%$ (57 of 294) before 2006 to $6.6 \%$ (25 of 380 ) after 2006 , in accordance with
TABLE 2. Reasons for mitral valve reoperation $(n=48)$

\begin{tabular}{lc}
\hline \multicolumn{1}{c}{ Reason } & n (\%) \\
\hline Recurrent mitral regurgitation from all causes & 39 \\
Prolapse (prolapse with ruptured artificial chordae) & $17(3)$ \\
Degenerative changes without prolapse & 19 \\
Mechanical problems of primary repair procedure & 3 \\
Hemolysis & 3 \\
Infective endocarditis & 4 \\
Other & 2 \\
Left ventricular pseudoaneurysm & 1 \\
Leaflet suture line dehiscence with mild MR & 1 \\
\hline$M R$, Mitral regurgitation. &
\end{tabular}

improvements in surgical skill and the quality of the intraoperative TEE assessment. Residual leaflet prolapse was found only in 5 patients $(0.7 \%)$. Thus, most residual MR had resulted from poor coaptation, despite the appropriate artificial chordal length. All 82 patients with residual MR had undergone serial follow-up TEE examinations. During the follow-up period, residual MR worsened in 39 patients $(47.6 \%)$, stayed unchanged in $29(35.4 \%)$, and improved in $14(17.1 \%)$.

\section{Long-Term Survival and Mitral Valve Reoperation}

The actuarial survival rate was $93.8 \%$ at 7 years and $85.9 \%$ at 12 years. The freedom from mitral reoperation rate was $96.4 \%$ at 7 years and $88.7 \%$ at 12 years. A total of 48 mitral reoperations were performed during the follow-up period. Three patients were found to have ruptured artificial chordae, and they underwent reoperation at 4,7 , and 84 months after the primary mitral valve repair. In all 3 cases, the rupture of the artificial chordae had occurred at the middle portion of the chordae, which was not the portion we had clamped during the primary surgery. We only repaired the mitral valve again in 7 patients and replaced the valve in 41 patients, mostly because of advanced degenerative disease. The reasons for the mitral reoperations are listed in Table 2.

\section{Late TTE Findings}

The follow-up TTEs showed that 94 of 674 patients had developed recurrent moderate or severe MR. The median interval to the recurrence of moderate or severe MR after surgery was 94 months (range, 0-227). Of those 94 patients, 33 had recurrent prolapse, 47 had degenerative changes without prolapse, 4 had infective endocarditis, and 10 had other pathologic findings, such as ring detachment, dehiscence of leaflet suture, and leaflet perforation. The freedom from recurrent moderate or severe MR rate was $82.6 \%$ at 7 years and $72.3 \%$ at 12 years (Figure 1). When stratified by the preoperative prolapse location, the 10-year freedom from recurrent MR rate was $63.5 \%$ in the anterior leaflet prolapse group, $81.1 \%$ in the posterior leaflet prolapse group, and $83.6 \%$ in the bileaflet prolapse group 


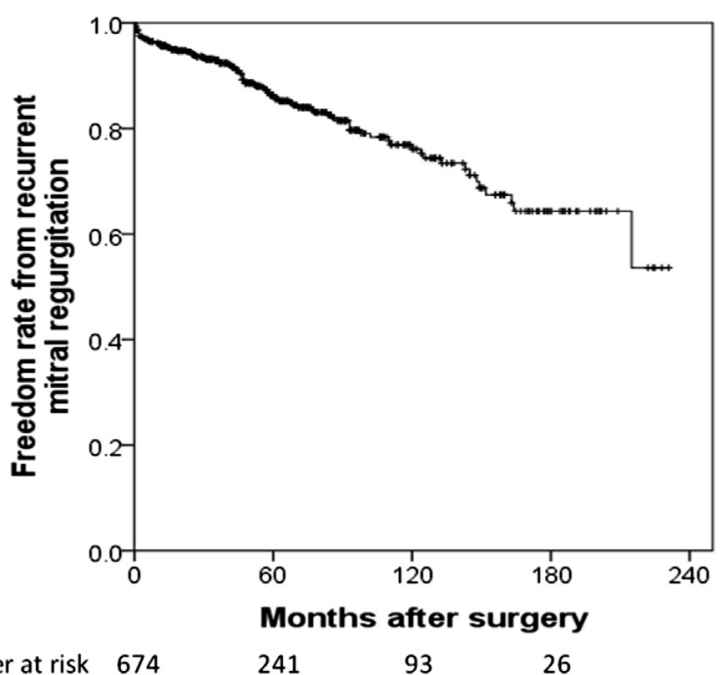

Number at risk 674

241

FIGURE 1. Kaplan-Meier curve for freedom from recurrent moderate or severe mitral regurgitation after mitral valve repair.

$(P<.001$; Figure 2$)$. The freedom from recurrent leaflet prolapse rate was $95.1 \%$ at 7 years and $89.0 \%$ at 12 years (Figure 3). Stepwise Cox regression analysis showed that the significant predictors of recurrent MR were anterior leaflet prolapse (HR, 2.0; 95\% CI, 1.1-3.6; $P=.016$ ), age (HR, $1.05 ; 95 \%$ CI, 1.02-1.07; $P=.001$ ), New York Heart Association class III or IV (HR, 1.8; 95\% CI, 1.1-2.9; $P=.026$ ), left ventricular end-systolic dimension (HR, $2.1 ; 95 \% \mathrm{CI}, 1.3-3.4 ; P=.004)$, no annuloplasty ring or band used (HR, 4.0; 95\% CI, 1.8-8.9; $P=.001$ ), and postoperative residual MR (HR, 5.2; 95\% CI, 3.2-8.6; $P<.001)$.

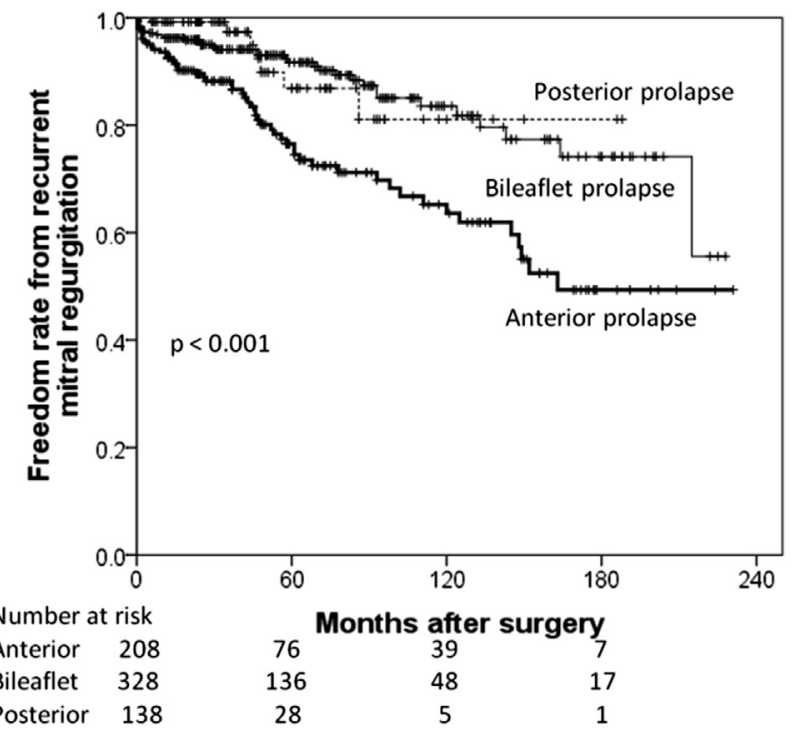

FIGURE 2. Kaplan-Meier curves for freedom from recurrent moderate or severe mitral regurgitation after mitral valve repair stratified by prolapse location.

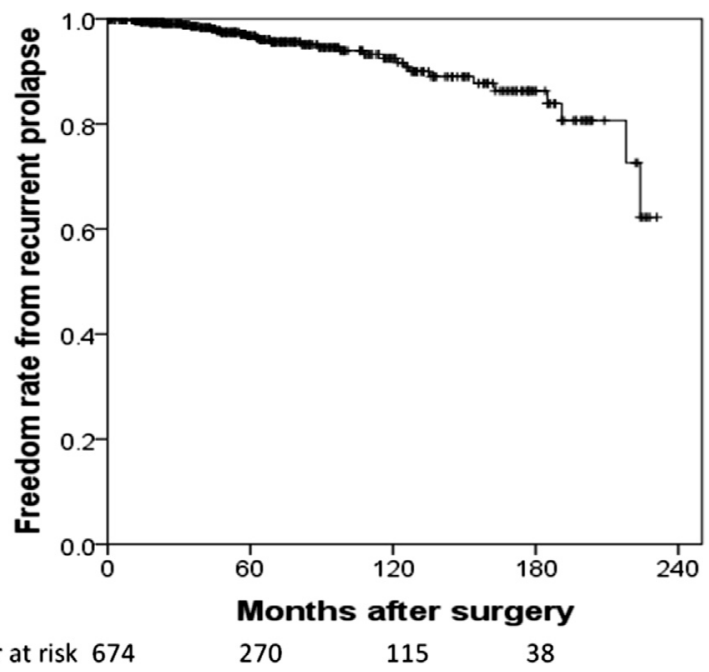

FIGURE 3. Kaplan-Meier curve for freedom from recurrent leaflet prolapse after mitral valve repair.

\section{DISCUSSION}

The tourniquet technique is a simple and reproducible method to determine the length of artificial chordae. We used this technique exclusively to adjust the chordal length during the study period. Our study has shown that this technique is effective and durable for repair of leaflet prolapse. One of the advantages of this technique is that it does not require echocardiographic or direct measurement of the chordal length. Many techniques using a fixed length of artificial chordae have been introduced. ${ }^{5-10}$ In those techniques, the fixed length is determined by preoperative echocardiographic measurement or intraoperative direct measurement using a caliper, both of which have a risk of measurement error. Also, the locations at which the sutures will be fixed on the papillary muscle and leaflet could be different from the points of measurement. In such cases, the fixed chordal length will not work well.

We have held the tourniquets at a specific length by reference to the normal adjacent chordae and readjusted the length after saline testing if the competency was not good. In some techniques, the chordal length will be determined by reference to the normal adjacent chordae without testing. ${ }^{11,12}$ A disadvantage of such techniques is that the chordal length cannot be corrected once a specific length has been obtained. The optimal length of the artificial chordae can be different from that of the adjacent normal chordae. Other techniques will create artificial chordae of adjustable length, such as in our technique. ${ }^{13-16}$ In those techniques, the artificial chordae will be held at a specific length using a temporary suture, a single loop or knot of the suture on the leaflet, hemoclips, and neurosurgical clips. Small light tourniquets will affect saline testing less than will large clips and have a lower risk of being lost in the chest than do small clips. Additionally, the chordal 
length can be easily adjusted by sliding the tourniquet tube up or down, which seems easier than undoing and redoing temporary sutures or knots. The advantage of the tourniquet technique is more apparent in minimally invasive procedures because all aspects can be controlled from outside the chest.

One of potential drawbacks of the tourniquet technique is that clamping ePTFE sutures might reduce their tensile strength. We found a ruptured artificial chorda in 3 mitral reoperation cases. Those ruptures could have been caused by intraoperative damage to the suture; however, none of those had ruptured at the site that had been clamped in the primary surgery. From our experience, gentle clamping of ePTFE sutures using a right-angled clamp seems safe; however, ePTFE sutures should be carefully handled during surgery to avoid damaging them. In the tourniquet technique, a right-angled clamp can be replaced by any other clamping devices such as a covered clamp, atraumatic vascular clamp, or neurosurgery clip without losing the advantages of this technique.

Another potential drawback is that the chordal length will be affected by the left ventricular volume at the saline test. If we fill the left ventricle too much, the chordal length would be longer than it should be. Artificial chords that are too long will cause residual prolapse or shallow coaptation. Thus, we need to monitor the left ventricular volume using TEE and determine the chordal length with the endsystolic left ventricular volume.

The results from the present study showed a low incidence of residual leaflet prolapse and recurrent leaflet prolapse after mitral valve repair using the tourniquet technique. Thus, the tourniquet technique is effective for fixing prolapsing leaflets and maintaining the leaflets in position. However, our residual MR (defined as mild or greater MR on the predischarge TTE) and recurrent MR (defined as moderate or severe MR on any follow-up TTE after discharge) rates were greater than the residual and recurrent prolapse rates.

The echocardiographic findings indicated that most residual MR had resulted from poor coaptation, despite repaired leaflet prolapse. The potential causes of residual poor coaptation included advanced degenerative changes in the leaflet coaptation zone and insufficient coaptation area. However, most of these cases of residual MR were not detected by intraoperative TEE. We also used to assess for residual MR partially on cardiopulmonary bypass, which could have underestimated the MR. Our threshold of using a second pump run for residual MR used to be $>2.0 \mathrm{~cm}^{2}$ of the regurgitant jet area, which seemed too optimistic. We have modified our intraoperative assessment protocol. Recently, we began measuring the coaptation length using the ink test ${ }^{17}$ and intraoperative TEE to confirm a sufficient postrepair coaptation area. Additionally, we assess residual MR with cardiopulmonary bypass off, and our threshold of a second pump run has been changed to $>1.0 \mathrm{~cm}^{2}$. These modifications and our technical improvement have decreased our residual MR rate.

On the follow-up TTEs, we found that the most common cause of recurrent MR was progressive degenerative changes of the leaflet without recurrent prolapse. In particular, in the anterior leaflet prolapse and bileaflet prolapse groups, this type of recurrent MR was often seen, and those valves were found to have developed remarkable thickening of the anterior leaflet and reduced mobility of the posterior leaflet. Also, most valves with recurrent prolapse were associated with progressive degenerative changes. The incidence of recurrent MR was greater in the anterior leaflet prolapse group than in the posterior leaflet and bileaflet prolapse groups, compatible with previously reported findings. ${ }^{18-20}$ No good method is available to control the progression of degenerative changes. However, deep coaptation at the primary mitral valve repair would seem very important to maintain sufficient coaptation, even after the leaflets have develop degenerative changes. Also, minimizing residual MR will be important to reduce the incidence of recurrent MR, because residual MR is a known predictor of recurrent $\mathrm{MR} .^{20}$

The present study had the limitations of an observational study. Because we did not compare the outcomes of the tourniquet technique with those of other techniques, we could not show the effect of this technique on the outcomes of mitral valve repair. In some patients, the MR group was evaluated semiquantitatively, which might have affected the robustness of the outcomes measures. However, quantitative measurements were not routinely performed in the early years of the study period, and some raw echocardiographic data were unavailable for measurement.

\section{CONCLUSIONS}

The tourniquet technique is a simple effective method to repair leaflet prolapse, with a low incidence of recurrent prolapse. The incidence of recurrent MR was high in the anterior leaflet prolapse group. Older age, no annuloplasty ring or band used, and residual MR were strong predictors of recurrent MR. Many cases of recurrent MR had resulted from progressive degenerative changes of the leaflet without recurrent prolapse.

\section{References}

1. David TE. Replacement of chordae tendineae with expanded polytetrafluoro-
ethylene sutures. J Card Surg. 1989;4:286-90.
2. Kasegawa H, Kamata S, Hirata S, Kobayashi N, Mannouji E, Ida T, et al. Simple
method for determining proper length of artificial chordae in mitral valve repair.
Ann Thorac Surg. 1994;57:237-8; discussion 238-9.
3. Bonow RO, Carabello BA, Chatterjee K, de Leon AC Jr, Faxon DP, Freed MD,
et al. ACC/AHA 2006 guidelines for the management of patients with valvular
heart disease: a report of the American College of Cardiology/American Heart
Association Task Force on Practice Guidelines (writing Committee to Revise
the 1998 guidelines for the management of patients with valvular heart disease)
developed in collaboration with the Society of Cardiovascular Anesthesiologists 
endorsed by the Society for Cardiovascular Angiography and Interventions and the Society of Thoracic Surgeons. J Am Coll Cardiol. 2006;48:e1-148.

4. Zoghbi WA, Enriquez-Sarano M, Foster E, Grayburn PA, Kraft CD, Levine RA, et al. Recommendations for evaluation of the severity of native valvular regurgitation with two-dimensional and Doppler echocardiography. J Am Soc Echocardiogr. 2003;16:777-802.

5. Von Oppell UO, Mohr FW. Chordal replacement for both minimally invasive and conventional mitral valve surgery using premeasured Gore-Tex loops. Ann Thorac Surg. 2000;70:2166-8.

6. Doi A, Iida H, Sunazawa T. Intracardiac calipers for artificial chordae replacement in mitral valve repair. Ann Thorac Surg. 2009;87:326-8.

7. Matsui Y, Fukuda Y, Naito Y, Sasaki S, Yasuda K. A new device for ensuring the correct length of artificial chordae in mitral valvuloplasty. Ann Thorac Surg. 2005;79:1064-5.

8. Mandegar MH, Yousefnia MA, Roshanali F. Preoperative determination of artificial chordae length. Ann Thorac Surg. 2007;84:680-2.

9. Calafiore AM, Scandura S, Iaco AL, Contini M, Di Mauro M, Bivona A, et al. A simple method to obtain the correct length of the artificial chordae in complex chordal replacement. J Card Surg. 2008;23:204-6.

10. Scorsin M, Al-Attar N, Lessana A. A novel technique of utilizing artificial chordae for repair of mitral valve prolapse. J Thorac Cardiovasc Surg. 2007;134: 1072-3.

11. Duran CM, Pekar F. Techniques for ensuring the correct length of new mitral chords. J Heart Valve Dis. 2003;12:156-61.
12. Chan DT, Chiu CS, Cheng LC, Au TW. Artificial chordae: a simple clip and tie technique. J Thorac Cardiovasc Surg. 2008;136:1597-9.

13. Fattouch K, Bianco G, Sbraga F, Sampognaro R, Ruvolo G. Simple, safe and easy technique to ensure the correct length of artificial chordae in mitral valve repair. Ann Thorac Surg. 2007;83:1902-3.

14. Okamoto K, Yozu R, Kudo M. Loop-in-loop technique in mitral valve repair via minithoracotomy. Ann Thorac Surg. 2012;93:1329-30.

15. Chocron S. Removable clips for mitral valve repair. J Thorac Cardiovasc Surg. 2007; 133:1682-3

16. Moorjani N, Viola N, Janusauskas V, Livesey S. Adjusting the length of artificial polytetrafluoroethylene chordae in mitral valve repair by a single loop technique. J Thorac Cardiovasc Surg. 2009;138:1441-2.

17. Anyanwu AC, Adams DH. The intraoperative "ink test": a novel assessment tool in mitral valve repair. J Thorac Cardiovasc Surg. 2007;133: 1635-6.

18. David TE, Armstrong S, Ivanov J. Chordal replacement with polytetrafluoroethylene sutures for mitral valve repair: a 25-year experience. J Thorac Cardiovasc Surg. 2013;145:1563-9.

19. Gillinov AM, Blackstone EH, Alaulaqi A, Sabik JF III, Mihaljevic T, Svensson LG, et al. Outcomes after repair of the anterior mitral leaflet for degenerative disease. Ann Thorac Surg. 2008;86:708-17.

20. Suri RM, Schaff HV, Dearani JA, Sundt TM III, Daly RC, Mullany CJ, et al. Survival advantage and improved durability of mitral repair for leaflet prolapse subsets in the current era. Ann Thorac Surg. 2006;82:819-26. 


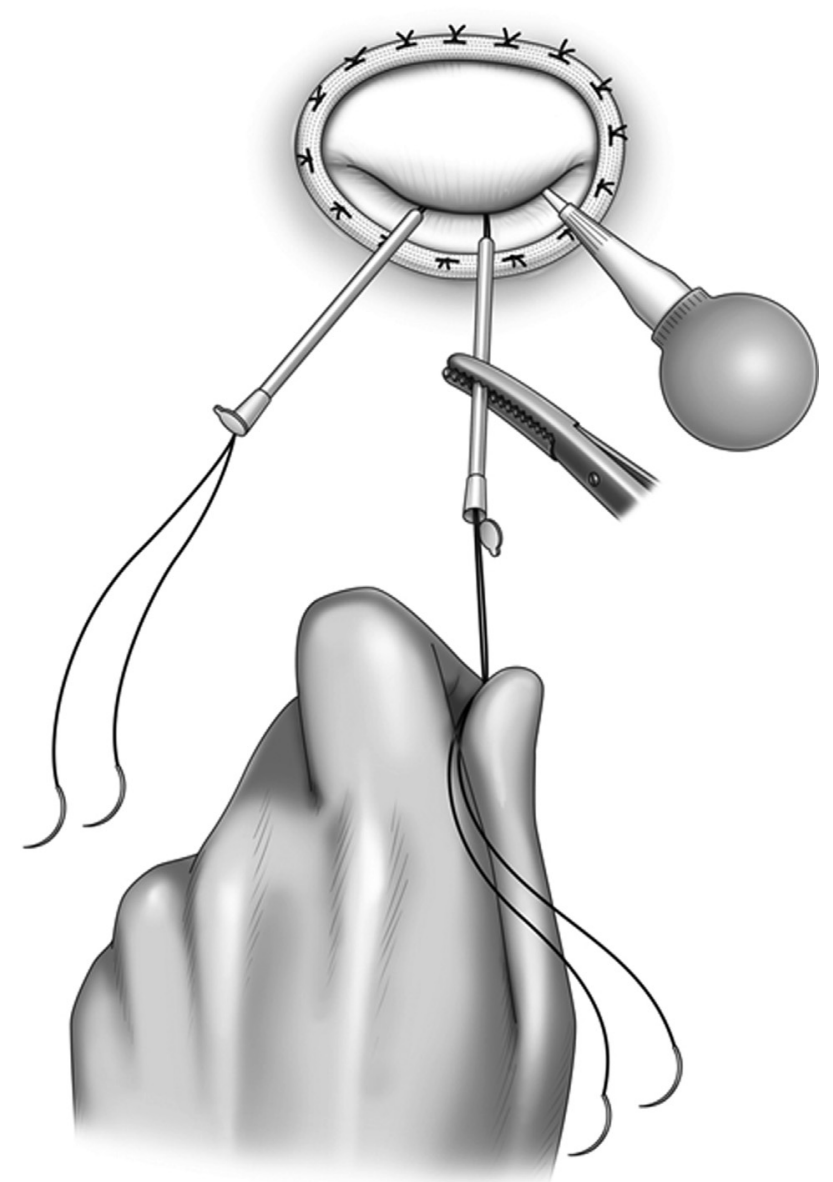

FIGURE E1. The tourniquet technique. Small tourniquets hold artificial chordae at a specific length temporarily, and competency is evaluated with saline testing.

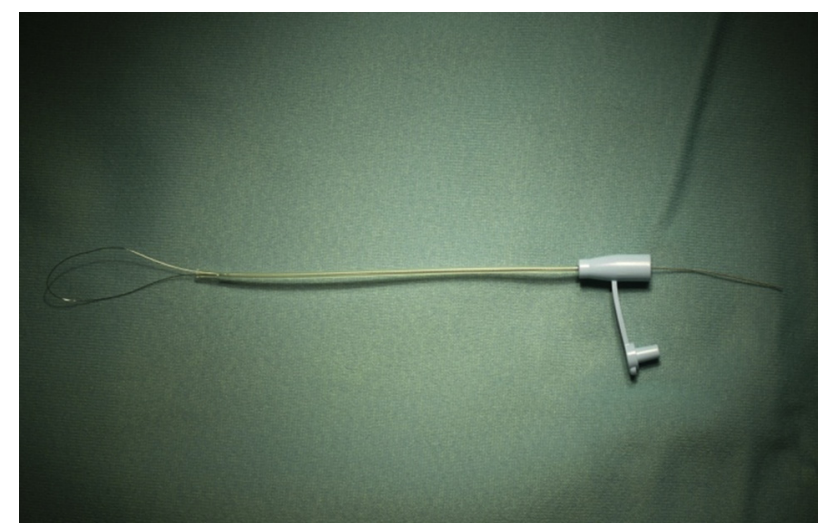

FIGURE E2. A tourniquet tube for the tourniquet technique. 